\title{
Recent twin revolutions in structural biology
}

\author{
M.R.N. Murthy \\ Molecular Biophysics Unit, Indian Institute of Science, Bengaluru 560012, Karnataka, India \\ Corresponding author: mrn@iisc.ac.in
}

\begin{abstract}
The development of optical microscopes led to the discovery of cells as the fundamental units of life. It made us aware that microbes dominate the living world. However, optical microscopes have limitation as to the size of cell and cell organelles they can focus. This size limit is referred to as the resolution of the microscope, which is in the order of $0.6 \lambda$, where $\lambda$ is the wave length of light. Wavelength of visible light ranges between $400 \mathrm{~nm}$ and $800 \mathrm{~nm}$ making the resolution of an optical microscope to the same order. Thus, organelles or the largest biomolecules cannot be seen. For example, ribosomes are about $\mathbf{3 0 0}$ times smaller when compared to the wavelength of visible light. To visualize such structures, electron microscopy and $\mathrm{X}$-ray diffraction are employed. Electron microscopy can magnify beyond visible ranges, enabling us to see organelles. $X$-ray diffraction has the power of revealing the atomic structure of molecules constituting the biological crystals. A revolution in X-ray diffraction using extremely intense $\mathrm{X}$-ray beams from synchrotrons developed by physicists for their high energy research became fundamental in structural biology. Synchrotrons provide $X$-ray beams that are several orders of magnitude higher in intensity compared to conventional $X$-ray sources. It is also possible to combine results of electron microscopy and $X$-ray diffraction to reveal structures of large viruses in a relatively short time. The secrets of life that $X$-ray crystallography and electron microscopy might unravel will undoubtedly constitute an exciting saga of twenty first century biology.
\end{abstract}

Keywords: X-ray diffraction, synchrotrons, electron microscopy, structural biology.

\section{INTRODUCTION}

From the beginning of human civilization, comprehension of the universe, discovery of the laws that govern natural phenomena and understanding of the nature of life have heavily relied on the empirical knowledge obtained through the sense organs. In particular, vision has been central to this understanding. However, our vision has its limitations. The eyes cannot clearly perceive objects smaller than about one tenth of a millimeter. Therefore, awareness of the existence of a vast microbial world inaccessible to our naked eyes but of crucial importance for our well-being had to await development of optical microscopes. High quality optical microscopes allow us to visualize or "see" bacterial cells. Optical microscopes firmly established that the fundamental unit of life is the cell and gave birth to the science of cell biology. It made us aware of the vast number and species of microbes that inhabit every inch of earth's surface.

However, optical microscopes also have their limitation. Images of very close objects with a separation smaller than a minimum value merge or overlap and hence cannot be perceived as distinct objects. This puts a limit on the size of the object that can be viewed using an optical microscope. This size limit is referred to as the resolution of the microscope. This limit is of the order of $0.6 \lambda$, where $\lambda$ is the wave length of light. Wavelength of visible light ranges between $400 \mathrm{~nm}$ and $800 \mathrm{~nm}$ and this sets the limit on the size of objects that can be distinctly seen in an optical microscope to the same order (400$800 \mathrm{~nm}$ ). Even the largest of biomolecules functioning in living cells are much smaller than this limit. For example, ribosomes that are central to the translation of the genetic information into functional protein molecules are about 300 times smaller when compared to the wavelength of visible light. Therefore, it is impossible to visualize biomolecules using a light microscope.

The bewildering complexity of living organisms is a collective, emergent property of the structure, function and interactions of tens of thousands of small and large biomolecules. Cellular function is determined by the three dimensional structures of these molecules. Therefore, determination of structures of these molecules is central to understanding of the function of the living cell 
and the nature of life. About a hundred years ago, single crystal (Figure 1) X-ray diffraction, the phenomenon of scattering of $\mathrm{X}$-radiation in specific directions by crystallize substances, was shown to have the power of revealing the atomic structure of molecules constituting the crystal.

\section{X-RAY DIFFRACTION}

X-rays are electromagnetic radiation of wavelength of the order of the distance between bonded atoms of molecules. When an X-ray beam hits a crystal, electrons of the atoms constituting the crystal scatter this radiation in all directions. However, because of the periodicity of atoms in the crystal, significant reflections occur only in certain directions along which the waves scattered from neighbouring cells of the crystal interfere constructively (the waves scattered by different unit cells are in phase). In other directions, the X-ray waves emanating from different cells add up to insignificant values. Therefore, scattering is limited to certain specific directions that depend on the direction of the incident beam and orientation of the crystal with respect to the beam (Figure 2).

As a technique of visualizing structures, X-ray diffraction differs drastically from optical microscopy as there are no lenses that can bend and focus X-rays. Therefore, image construction in X-ray diffraction has to be carried out by the experimentalist. This process involves determination of the relative phases of reflections and very large-scale computations. The differences between light microscopy and structure determination by diffraction technique is illustrated in Figure 3.

\section{SYNCHROTRONS}

X-rays are electromagnetic radiation of wavelength 1 $10 \AA$ (Angstrom, one tenth of a nanometer). CuKa radiation (wavelength $1.5418 \AA$ ) is produced by $X$-ray generators equipped with a copper anode when an electron beam accelerated by a critical voltage (about 9000 volts, although in practice $40,000-60,000$ volts are used to compensate for the loss of energy upon collision with the thin atmosphere of the evacuated X-ray tube) hits the copper anode. More intense beams of X-rays are obtained from rotating anode $X$-ray generators where the anode is rotated to minimise heating. A revolution in $\mathrm{X}$ ray diffraction data collection took place when, in the 1980s and 1990s, extremely intense X-ray beams from synchrotrons developed by physicists for their high energy research became available for structural studies on biological macromolecules. In synchrotrons, electrons go around a closed, nearly circular evacuated path of about a kilometre circumference at speeds that are close to that of light. When electrons bend from straight paths due to the magnetic field of powerful magnets inserted in the ring, they emit intense X-radiation over a wide wavelength range. Synchrotrons provide X-ray beams that are several orders of magnitude higher in intensity compared to conventional X-ray sources.

Most of structural studies on biological macromolecules are now carried out using synchrotron radiation. This has reduced the diffraction data recording time from months or years to hours. Recently, a synchrotron has become operational at Indore, Madhya Pradesh in our country. Another challenging problem that was encountered in early studies on structure of proteins was crystal decay caused by exposure to the ionizing $\mathrm{X}$-radiation. This has largely been overcome by cooling the crystals to liquid nitrogen temperatures during data collection. At such low temperatures, the ions and free radicals produced by bombarding $X$-rays are immobile and hence do not cause much damage to the crystal. During the past three decades, the rather insensitive photographic films used earlier for recording X-ray diffraction data have been replaced by imaging plate $\mathrm{X}$-ray detectors and CCD cameras. These are much more sensitive detectors, possess a much larger dynamic range (capable of measuring very small and very large intensities) and are prone to much less errors. Structure determination by X-ray diffraction involves large scale computation. Fortunately, computers have become much more powerful and much less expensive over the past several decades.

All these developments have resulted in an explosion in the number of structures determined. It has made $X$ ray diffraction the most powerful tool for the elucidation of macromolecular structures. This field has revolutionized chemistry and biology by allowing determination of the atomic structure of hundreds of thousands of molecules. Apart from the structures of organic and inorganic compounds, structures of enzymes, toxins, carrier molecules, antibody molecules, hormones, receptors, signalling proteins, viral structural and non-structural proteins, "molecular machines" such as fatty acid synthase multienzyme complex, photosynthetic reaction centre, ATP synthase, several large virus particles and ribosome etc. have been determined suing single crystal X-ray diffraction techniques. There is hardly any branch of biological science that has not been profoundly influenced by X-ray crystallography. The structure of DNA forms the backbone of molecular and cellular biology. The mechanisms by which the information on DNA is transferred to RNA and from RNA to proteins has been deduced from structural studies on hundreds of proteins involved in transcription and translation.

Structural studies on antibodies and antigen-antibody complexes has provided the molecular basis for understanding immunology. It has been possible to understand the complex mechanisms by which polar molecules get transported across the semi-permeable biological membranes by structural studies on ion channels and other transport proteins. Thousands of structural studies on protein enzymes have provided detailed information on 
Figure 1: Crystals of one of the mutants of a protein induced in Salmonella typhimurium when the bacterium is subjected to environmental stress.

Figure 2: X-ray diffraction photograph recorded on a crystal of a phosphate binding protein of Stenotrophomonas maltophilia involved in phosphate transfer across bacterial cell membrane.

Figure 3: Comparison of optical microscopy and X-ray crystallography. Left side shows the arrangement in an optical microscope where the image is formed by the bending of light by lenses. Rest of the picture illustrates the method of obtaining image of a molecule in Xray crystallography. Intensities of X-ray reflections caused by a crystal contain information about the structure of the molecule constituting the crystal. However, an "electron density map" representing an image of the molecule can be obtained only after determining the relative phases of the reflections followed by extensive computation.
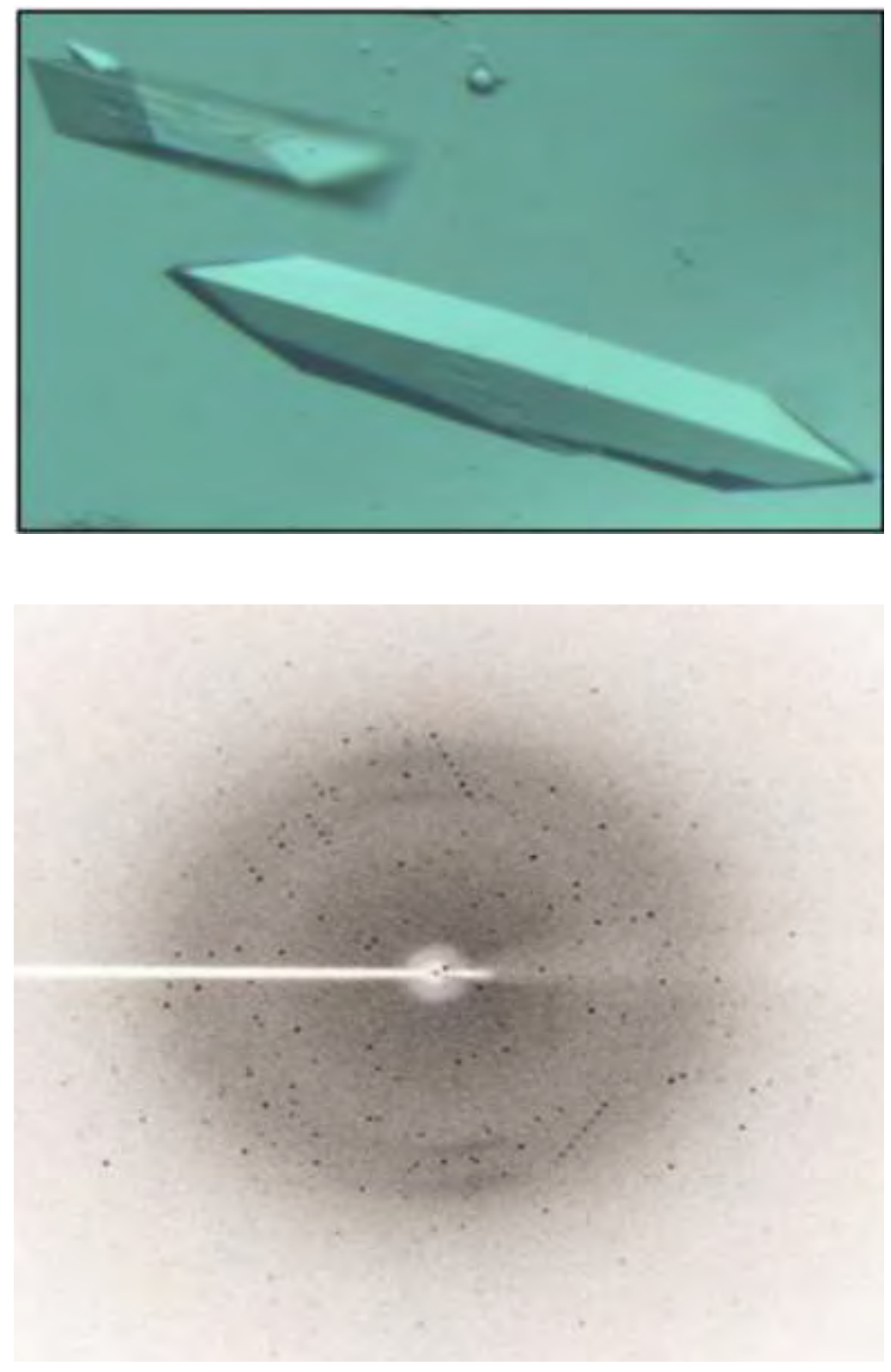

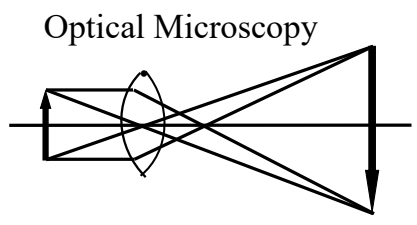

Light scattered by the object is focussed on the image plane resulting in an enlarged image
Diffraction pattern recorded on a film

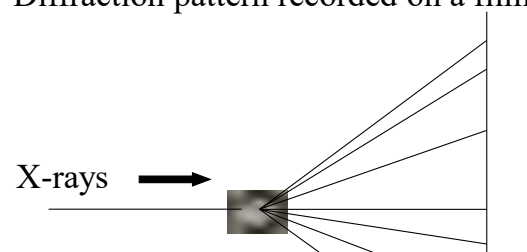

Crystal scatters X-rays

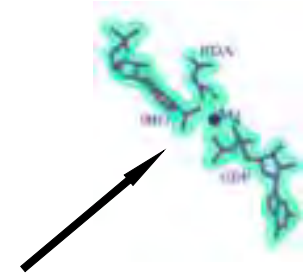

An electron density map representing An image of the object is computed

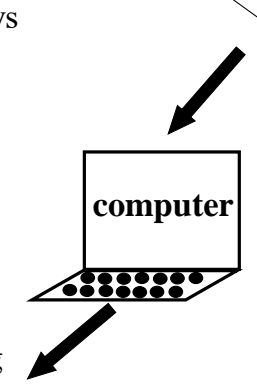


key properties such as efficiency of enzyme catalysis that take place at ambient temperature, enzyme regulation, feedback inhibition, allosteric properties etc. These properties of protein control the metabolic state of cells. Gene regulation, mechanism of invasion and cell entry by pathogens, structural aspects of cell differentiation, regulation of cell division and mutations that might lead to the onset of cancer are some of the other aspects that have been investigated in structural terms.

On protein structural aspects, $\mathrm{X}$-ray diffraction studies have revealed the patterns of protein folding, preferred spatial organisation of secondary structural elements, modular organisation of proteins in to spatial domains and molecular details of protein-protein, protein-nucleic acid and protein-ligand interactions, the effects of mutations, the mechanisms of protein evolution and functional specialisation of proteins. X-ray crystallography has not only provided deep understanding of molecular processes that define cellular function but also has made possible novel biotechnological applications. These include protein engineering to improve enzyme function, stabilizing protein folds and drug design against diseases caused by pathogens. The number of macromolecular structures determined so far using X-ray diffraction exceeds 150,000 . These structures are deposited in the protein data bank and are freely accessible to all biologists.

\section{ELECTRON MICROSCOPY}

Structure determination by X-ray diffraction technique needs crystals of the material or molecule whose structure needs to be determined. Electron microscopy has recently emerged as a powerful, alternative tool for structural studies on biological macromolecules that cannot be crystallized as well as for molecules that are too large for X-ray diffraction studies. The technique of elec- tron microscopy has a long history although it has become a remarkably powerful tool for the elucidation of biomolecular structures only over the past three decades. In 1924, the French physicist de Broglie suggested that electrons, till then considered as particles encircling atomic nuclei, could behave like waves. He suggested a formula for the wavelength of the waves associated with electrons.

$$
\lambda=0.1(150 / V)^{0.5}
$$

Where $V$ is the voltage applied in Volts and $\lambda$ is the wavelength in Angstroms. Thus, higher the applied voltage, lower the wavelength associated with the electron wave. Accelerating electrons by 100,000 volts results in a wavelength of approximately $0.122 \AA$.

Immediately after de Broglie proposed his theory of electron waves, several scientists realized that it should be possible to construct a microscope using electron waves instead of light waves and that would overcome the limitation on resolution set by the wavelength of light. However, it was known that electrons interact strongly with all matter and constructing a microscope needed resolution of several technical problems. In spite of these difficulties, Ruska and Knowl succeeded in constructing the first electron microscope in 1932. However, the successful exploitation of the microscope was very slow in coming due to innumerable problems such as the destructive nature of electrons when they interact with samples. Therefore, recognition for Ruska's work came almost fifty years later, in 1986, when he was awarded the Nobel Prize. A picture of the first electron microscope built by Ruska and downloaded from the web is shown in Figure 4.

Because electrons cannot travel in air, the whole inner chamber of the electron microscope has to be evacuated to at least one-millionth of atmospheric pressure.
Figure 4: Electron microscope built by Ruska and Knowl.

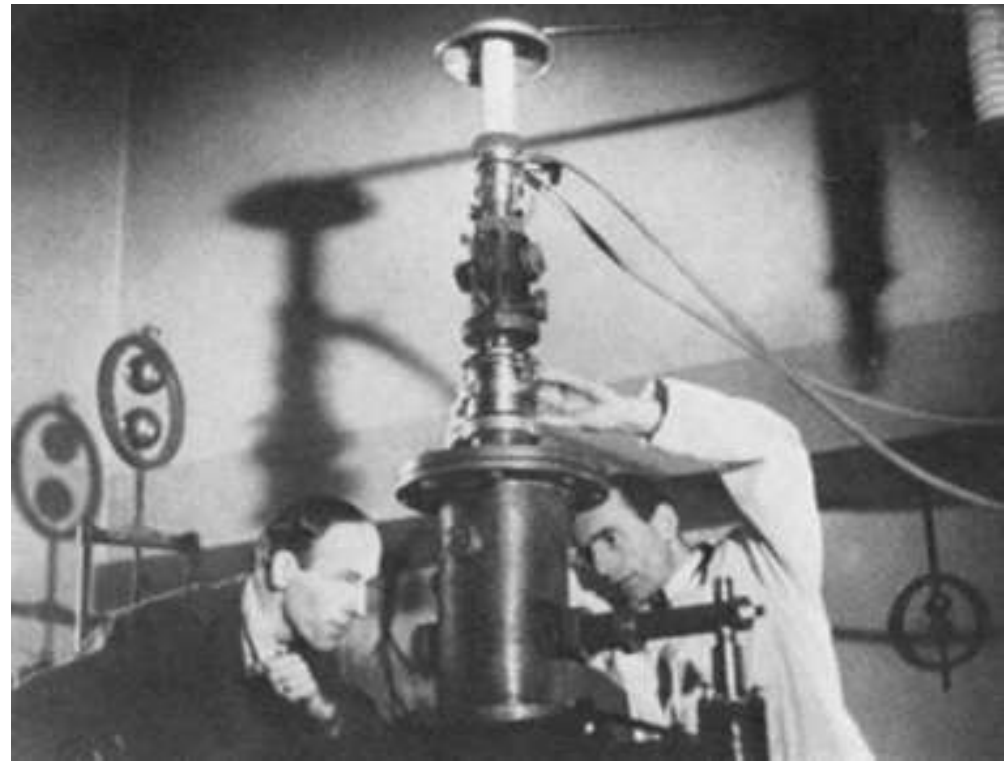


Electrons are emitted by an electron gun, which could be a heated tungsten filament or a field emission gun. The released electrons are accelerated by the application of a positive potential. Electro magnets act as lenses and bend or focus the electron waves. Unlike the fixed focal length of lenses in optical microscopes, the focal lengths of electromagnetic lenses can be continuously varied by changing the current flowing through the coils of the electro magnet. A system of condenser lenses focusses the electron beam on to the sample. Another series of objective, intermediate and projector leases focus the image on to the image plane. However, before electron microscope became practically useful, each of these systems had to undergo a great deal of modification, advancement and refinement.

Since almost all materials are opaque to electrons, the sample for electron microscopic examination has to be thin and needs to be deposited on the so called "holey grids" (Figure 5). These are thin copper plates containing an array of small holes. These grids are coated with a thin layer of carbon. A drop of the solution containing the sample to be examined is loaded on to the grid and the particles is allowed to bind to the carbon layer for a few seconds. The rest of the drop is removed by absorbing the remaining liquid with a filter paper. Examination of such a grid in the electron microscope suffers from lack of contrast as the scattering of electron waves by the sample and the background carbon film are of the same order. This is like trying to see a thin glass plate immersed in water. The technique to increase the contrast that was developed in the fifties is to coat the sample by a layer of a heavy metal such as phosphotungstic acid which strongly scatters electrons. After binding the desired molecule to the grid, a drop of a solution containing the heavy metal salt is loaded on to the grid. Excess solution is removed by a filter paper. The grid is then visualized in the electron microscope. Because the scattering by the heavy metal salt is much higher than that of the sample, the contour of the sample appears bright in the dark background of the metal layer (Figure $6)$. This procedure has been called "negative staining technique".

Negative staining technique was extensively used in the 50 s and 60 s to study the architecture of several viruses. It was possible to 'see' tobacco mosaic virus (TMV) particles as rigid rods for the first time using an electron microscope. Figure 6 illustrates electron micrograph of a plant virus, Sesbania mosaic virus. The architectural details of several viruses that were spherical in appearance could be worked out. Viruses appeared to follow the construction principles developed by the architect Buckminster fuller for the construction of geodesic domes.

Although the successes of using electron microscopy to understand biomolecules, in particular the principles of viral architecture that were learnt in the 50 s and 60s were exciting, the limitation of electron microscopic examination of negatively stained specimens became evi- dent soon. Preparation of sample for electron microscopic examination required the sample to be dehydrated (dried) and stained with a heavy atom salt. This distorted the molecule constituting the sample and limited the resolution of the images.

\section{CRYO-ELECTRON MICROSCOPE}

To keep the samples hydrated and yet prevent dehydration in the vacuum chamber of the microscope needed controlling the temperature of the sample to very low values; in the range of -130 to $-180^{\circ} \mathrm{C}$. Cooling the sample to this low a temperature invariably resulted in the formation of ice crystals that distorted the structure of the molecule to be visualized. A protocol that resulted in transformation of liquid water in to an amorphous phase (non-crystalline) had to be developed. By trial and error, it was realized that transferring the grid to liquid ethane first followed by transfer to liquid nitrogen overcomes the problem of ice formation. Specialized equipment for this serial transfer to liquid ethane and liquid nitrogen were developed. These developments allowed visualization of hydrated samples in which the native structure of biomolecules was retained. Electron microscope also had to be developed to maintain cryogenic temperatures in the sample chamber. Figure 7 shows photograph of an early version of cryo-electron microscope.

The problem of low contrast is very serious with hydrated samples. The scattering by the sample is only a small fraction of the unscattered waves and hence the image is nearly invisible. Adequate information for a clear image is not contained in the amplitudes of scattered waves. However, the scattering process introduces a phase shift of $90^{\circ}$. Therefore, the unscattered and scattered beams are not in phase. The phase difference represents the image much better than the changes in amplitude across the image plane. This phase difference creates contrast if the image is slightly defocused. Thus, at the exact focal plane, the object is nearly invisible. When the image plane is set at a slight under focus, the contrast increases and the object becomes visible. However, this defocusing necessitates a correction to the image. Electron micrographs represent two dimensional projections of three-dimensional structures. If the particles are randomly oriented, images of tens of thousands of particles provide projection of the sample in almost every possible direction. In principle, it should be possible to combine these thousands of two-dimensional images to obtain the three-dimensional structure of the object. However, this necessitates determining the direction of projection for each particle image. The mathematical and computational methods required for reconstruction of three-dimensional images from a large number of two dimensional projections has been developed as a result of extensive efforts of several groups.

It is also possible to combine results of electron mi- 
Figure 5: Holey grids used to provide the support base for mounting specimens for electron microscopic observations. The diameter of the grid is about 3 millimetres.

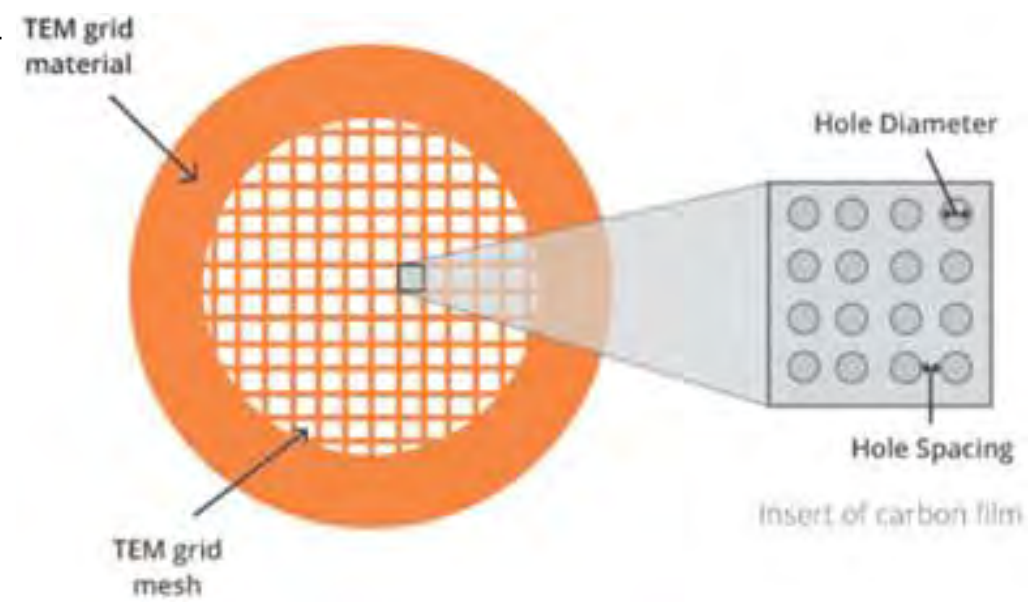

Figure 6: Electron micrograph of a plant virus, Sesbania mosaic virus, obtained using the negative staining technique. The diameter of the particles is about $30 \mathrm{~nm}$. The threedimensional structure of this virus has been determined by X-ray diffraction techniques.

Figure 7: An early version of cryo-electron microscope that has been installed at the Indian Institute of Science, Bengaluru. The sample chamber is maintained at liquid nitrogen temperature. Much more sophisticated cryoelectron microscopes are now available.
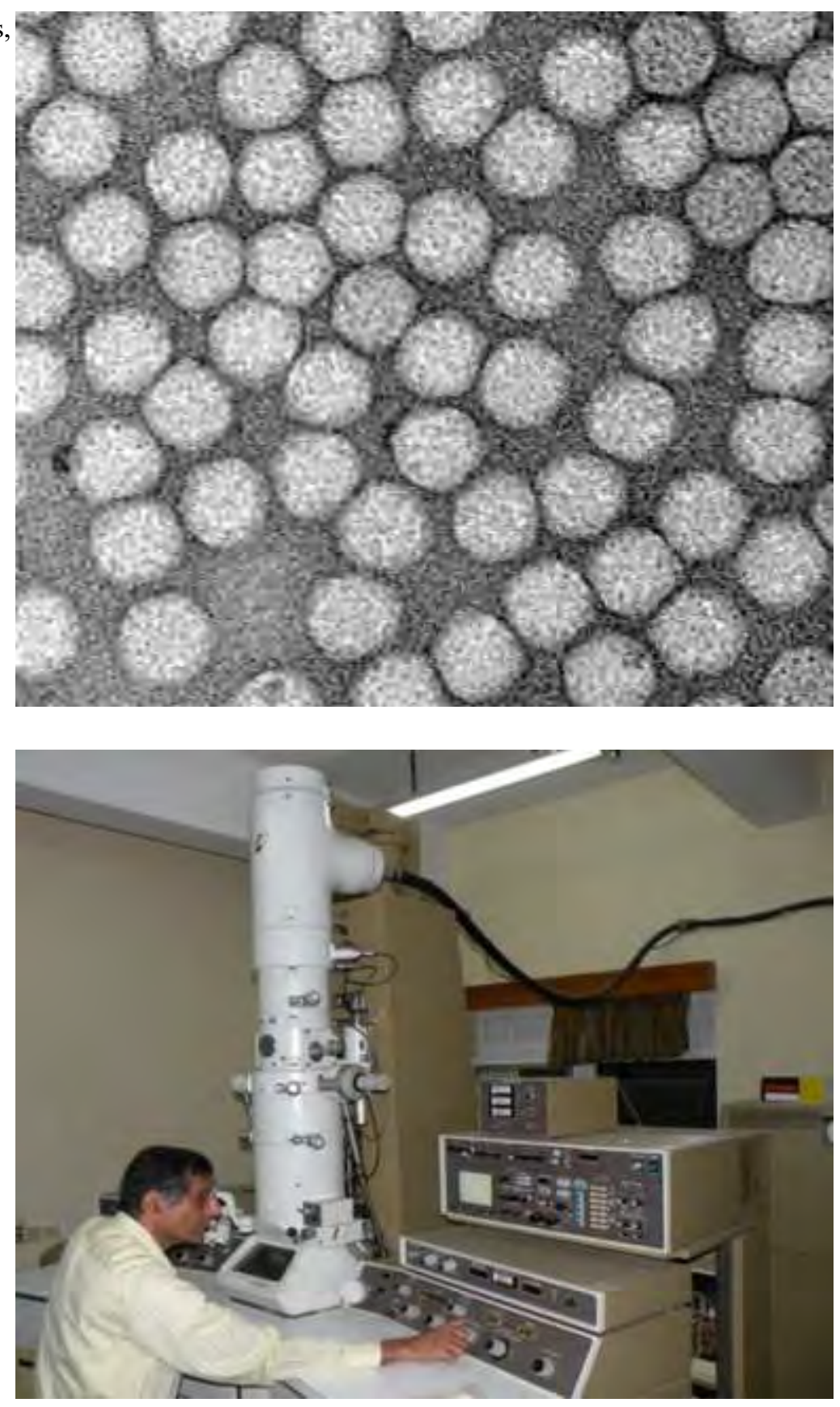
croscopy and X-ray diffraction to reveal structures of large viruses in a relatively short time. Virus particles are made up of a large number of identical protein subunits or a large number of a limited number of different protein molecules. If the protein molecule could be crystallized, its high-resolution structure could be obtained using X-ray crystallographic methods. A low-resolution image of the large virus particle could be obtained by electron microscopy. Copies of protein molecules could then be fitted into three-dimensional reconstruction obtained from electron microscopy and the structure of the entire virus particles could be obtained at high resolution. This method has been used to obtain the structure of Zika virus.

Cryo-electron microscopy has already become an extremely powerful tool for studying biomolecular structure and function. The power of this technology will certainly be further enhanced by technical advances in sample preparation, optical systems of the microscope, improving the contrast of images, detector technologies and mathematical methods of analysis. The secrets of life that X-ray crystallography and electron microscopy might unravel will undoubtedly constitute an exciting saga of twenty first century biology.

\section{REFERENCES}

Bai, X. C., McMullan, G., Scheres, S. H. (2015). How cryo$\mathrm{EM}$ is revolutionizing structural biology. Trends in Biochemical Sciences, 40(1): 49-57.

Carroni, M., Saibil, H. R. (2016). Cryo electron microscopy to determine the structure of macromolecular complexes. Methods, 95: 78-85.

Fernandez-Leiro, R., Scheres, S. H. (2016). Unravelling biological macromolecules with cryo-electron microscopy. Nature, 537(7620): 339-346.

Garman, E. F. (2014). Developments in x-ray crystallographic structure determination of biological macromolecules. Science, 343(6175): 1102-1108.

Orlov, I., Myasnikov, A. G., Andronov, L., Natchiar, S. K., Khatter, H., Beinsteiner, B., Ménétret, J.F., Hazemann, I., Mohideen, K., Tazibt, K. and Tabaroni, R. (2017). The integrative role of cryo electron microscopy in molecular and cellular structural biology. Biology of the Cell, 109(2): 81-93.

Polcari, D., Dauphin-Ducharme, P., Mauzeroll, J. (2016). Scanning electrochemical microscopy: a comprehensive review of experimental parameters from 1989 to 2015. Chemical Reviews, 116(22): 13234-13278.

Shi, Y. (2014). A glimpse of structural biology through Xray crystallography. Cell, 159(5): 995-1014.

Zhu, Y., Zhang, J., Li, A., Zhang, Y., Fan, C. (2017). Synchrotron-based X-ray microscopy for sub-100 nm resolution cell imaging. Current Opinion in Chemical Biology, 39: 11-16. 